\title{
Experimental Observation of IFEL Micro-Bunching Using Coherent Transition Radiation
}

\section{Y. Liu and D.B. Cline, University of California, Los Angeles, CA 90095}

X.J. Wang, M. Babzien, J.M. Fang, J. Gallardo, K. Kusche, I. Pogorelsky, J. Skaritka, A. van Steenbergen, Brookhaven National Laboratory, Upton, NY 11973

\section{Abstract}

Electron beam bunching in the optical wavelength was observed experimentally for the first time at the Brookhaven Accelerator Test Facility(ATF) using the Inverse Free Electron(IFEL) accelerator. The micro-bunched electron beam has been studied by measuring the coherent transition radiation (CTR). We have experimentally observed a quadratic dependency of the CTR signal with the charge of the electron beam and the observation distance.

\section{INTRODUCTION}

One of the major challenges in laser accelerator research is to generate a electron beam with a bunch length shorter than the laser wavelength for efficient acceleration and small energy spread. Several techniques[1,2] were proposed to produce micro-bunched electron beam for laser accelerator applications. A UCLA-BNL collaboration recently has experimentally demonstrated electron beam micro-bunching on the order of a few micron using the IFEL accelerator[3]. Operating the IFEL as a laser accelerator buncher has several advantages. Since IFEL does not involves any medium, such as plasma based laser accelerators, there is negligible electron beam quality degradation. Secondly, there is natural synchronization between the IFEL buncher and laser accelerator since the same laser will be used for both bunching and acceleration. We will first briefly discuss the coherent transition radiation properties, then the experimental setup will be described. The experimental result will be presented in the second half of the report.

\section{COHERENT TRANSITION RADIATION}

Coherent transition radiation is a collective effect of transition radiation produced by a large ensemble of electrons being in phase with each other. The intensity of photon radiated is highly enhanced and the total radiation distribution becomes[4]

$$
\frac{d^{2} U}{d \omega d \Omega}=\left.[N+N(N-1) F(\omega, \theta)] \frac{d^{2} u}{d \omega d \Omega}\right|_{\text {single }},
$$

where

$$
F(\omega, \theta)=\left|\iiint f(r, z) \exp (-i \vec{k} \cdot \vec{x}) d^{3} x\right|^{2}
$$

is a bunching factor, containing information about the electron distribution. The coherent effect scales like $N^{2}$ compare to the incoherent part, which scales linearly with the electron number, $N$. For a single foil case the CTR angular distribution has been estimated by J. Rosenzweig, et al[5].

For a two-foil configuration (Figure 1), the first foil is perpendicular to the electron beam direction, contributing a forward CTR, and the second foil is at $45^{\circ}$ to the electron beam direction, contributing a backward CTR. The individual electrons distributed on the second foil will have an additional phase difference $\varphi(x, L)$ relative to the electron on the first foil. The phase difference is given by
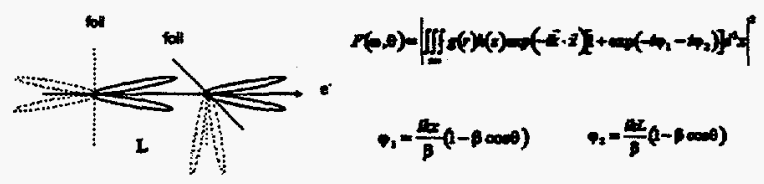

Figure 1: Two-foil configuration. First one generates a forward CTR and redirected by a second foil; the second foil generates backward CTR.

$$
\begin{aligned}
\varphi(x, L) & =\varphi_{1}(x)+\varphi_{2}(x, L) \\
& =\frac{k x}{\beta}(1-\beta \cos \theta)+\frac{k L}{\beta}(1-\beta \cos \theta),(3)
\end{aligned}
$$

where $L$ is the separated distance between the two foils. Following the same treatment for a single foil, the two-foil CTR photon angular distribution is expressed as follow:

$$
\begin{aligned}
\frac{d N_{p h}}{d \theta} \approx & \frac{\alpha \beta^{2}}{8 \sqrt{\pi^{3}}}\left(\frac{k_{r} \sigma_{z}}{\pi}\right)\left(\pi \frac{N b_{n}}{k_{r} \sigma_{z}}\right)^{2}\left(\frac{1}{n}\right) \frac{\sin ^{3} \theta}{(1-\beta \cos \theta)^{2}} \times \\
& \left\{\exp \left[-\left(\frac{n k_{r} \sigma_{r}}{\beta}\right)^{2}(\beta \sin \theta-\beta \cos \theta+1)^{2}\right]+\right. \\
& \exp \left[-\left(n k_{r} \sigma_{r} \sin \theta\right)^{2}\right]+ \\
& 2 \exp \left[-\frac{1}{2}\left(\frac{n k_{r} \sigma_{r}}{\beta}\right)^{2}(\beta \sin \theta-\beta \cos \theta+1)^{2}\right] \times \\
& \exp \left[-\frac{1}{2}\left(n k_{r} \sigma_{r} \sin \theta\right)^{2}\right] \times \\
& \exp \left[-\left(\frac{L}{2 \beta \sigma_{z}}\right)^{2}(\beta \cos \theta-1)^{2}\right] \times \\
& \left.\cos \left[\left(\frac{n k_{r} L}{\beta}\right)(\beta \cos \theta-1)\right]\right\}
\end{aligned}
$$

From the above equation we see that the total CTR intensity is produced by two individual CTR sources plus their interference in the far region. 


\section{EXPERIMENT SETUP}

In the current condition the maximum charge delivered to the IFEL wiggler at ATF is up to $300 \mathrm{pC}$. The space charge effect on the IFEL self-bunching can be ignore[6]. The basic experimental setup can be described as follows. A target (a component to generate CTR) is mounted inside a 6-way cross. The 6-way cross serves as a small vacuum chamber and is able to move along the beam path to find the optimum bunching position during the measurement. Thus, the 6-way cross connected with a pair of bellows is inserted into the beamline. This allows the target to travel back and forth along the $e^{-}$beam path without disturbing the vacuum environment. The radiation is transported from the chamber through a $\mathrm{ZnSe}$ window and a IR lens into a collecting system, collected by a parabolic mirror and focused into a detector (Figure 2). The detec-

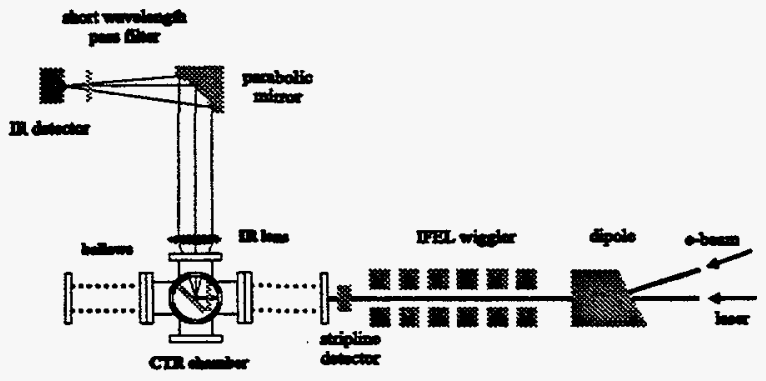

Figure 2: Schematic of the micro-bunching diagnostic setup for the IFEL experiment.

tor, liquid Nitrogen cooled, is sensitive to wavelengths from $1.1 \mu \mathrm{m}$ to $5.5 \mu \mathrm{m}$ with $1 \times 1 \mathrm{~mm}^{2}$ sensitive area; it is located $60 \mathrm{~cm}$ away from the target chamber and is well shielded by lead bricks. A HeNe laser is used for optical alignment and parabolic mirror rotation monitoring. For convenience, all components including the target chamber are assembled on a translated stage (movable table) driven by a digitized remote-controlled stepping motor. This allows the whole diagnostic system to travel along the $e^{-}$beam path for a maximum distance of $40 \mathrm{~cm}$.

Such a design keeps the optical alignment from changing when the target is moved. A stripline detector is placed between the upstream bellows and the exit of the wiggler to provide the total $e^{-}$beam charge information before it strikes the target. The output amplitude of the stripline detector is proportional to the total charge $(e N)$, where $e$ is the electron charge and $N$ is the number of electrons. Control and data collection are performed at the ATF control room.

\section{EXPERIMENTAL RESULTS}

The wiggler magnetic field steering effect on the $e^{-}$beam was investigated by using a high sensitivity $C C D$ camera to look at the optical transition radiation emitted from the surface of the foil (target). When the wiggler magnetic field
$(B)$ is strong, which matches with a high energy $e^{-}$beam, the $e^{-}$beam is strongly focused and steered away from the target. The situation is greatly improved after an additional steering magnet is placed right after the exit of the wiggler. Backgrounds were studied during the CTR measurement. There are three types of backgrounds. 1) $x$-rays generated by the $e^{-}$beam scattering. 2) broad bandwidth radiation produced the $\mathrm{CO}_{2}$ laser when it passes through the sapphire waveguide in the wiggler. 3) incoherent transition radiation combined with the coherent transition radiation. The backgrounds were measured under the same conditions as the CTR measurement, except that the laser electric field has no spatial overlap with the $e^{-}$beam (by changing relative timing delay), which ensures that there is no acceleration (no bunching). Thus, all combined three backgrounds are measured. However, the total background amplitude is much smaller than the CTR intensity amplitude (Figure 3).

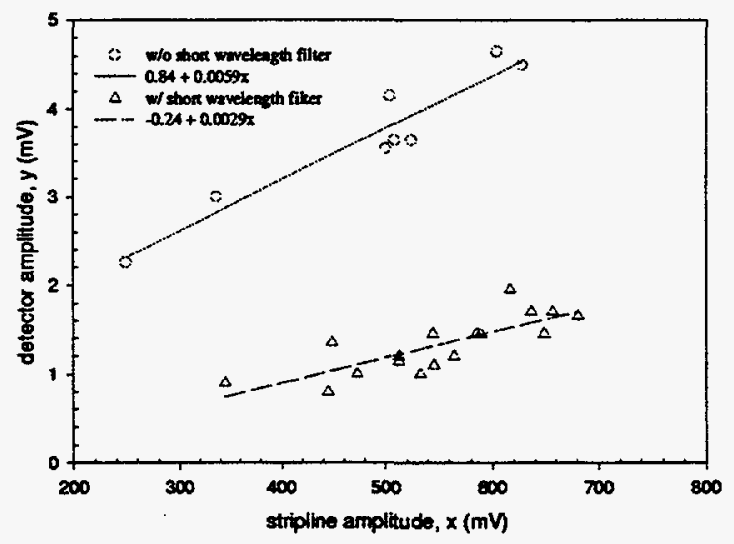

Figure 3: The circles represent the background taken without a short wavelength pass filter. The uptriangle is with the filter.

The CTR intensity amplitude as a function of the $e^{-}$ beam charge was measured. The results are plotted in Figure 4. Since the cutoff wavelength of the liquid Nitrogen cooled Indium Antimonide (InSb) detector is at $5.5 \mu \mathrm{m}$ and the detected CTR intensity is relatively strong, this shows that the microbunch length should be less than $5.5 \mu \mathrm{m}$. Furthermore, a short wavelength pass filter having a cutoff wavelength at $2.6 \mu \mathrm{m}$ was placed in front of the detector. The background and CTR data are plotted in Figure 5. At such a short wavelength region, the detector efficiency drops to less than $50 \%$, but the CTR is still detectable. This is definite proof that the bunch length is much shorter than $5 \mu \mathrm{m}$ and close to the fourth harmonic wavelength.

Last, the micro-bunch length change as a function of its drifting distance is examined by positioning the target at different locations (Figure 6). The distance is defined from the wiggler exit to the target position. As predicted, after optimum bunching distance the microbunched electrons start to debunch (bunch length increase) and results in the 


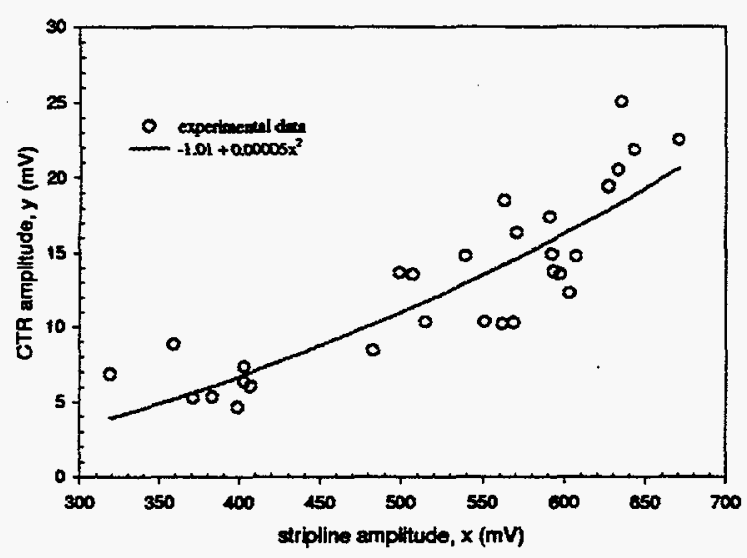

Figure 4: The total CTR intensity amplitude as a function of the total number of electrons. The stripline detector output amplitude is proportional to the total charge $(e N)$.

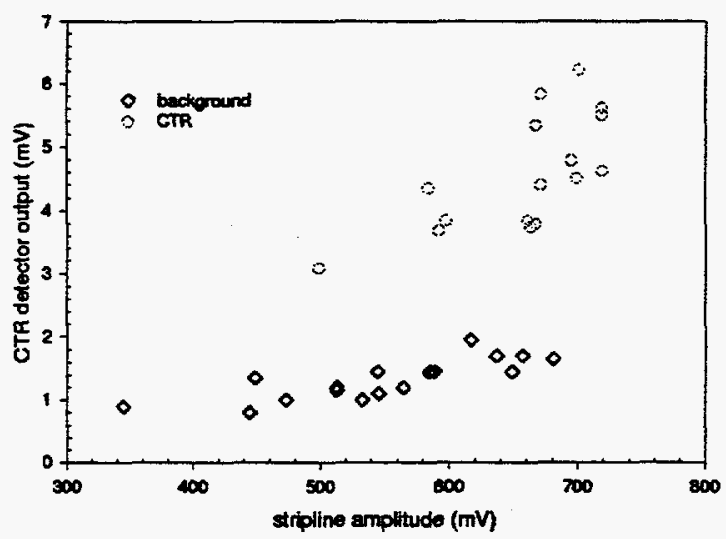

Figure 5: The detected CTR intensity amplitude is well above the background amplitude at short wavelength region. A short wavelength pass filter is used during the measurement.

CTR intensity amplitude decrease. The experimental data shown in Figure 6 reasonably agree with the model prediction. The simulation results[7] show that the optimum microbunch length (FWHM) is less than $2 \mu \mathrm{m}$.

Note, only the debunching process was studied. This is due to the following facts: (1) space-charge may affect the self-bunching process even when the charge density is not very high. This may lead to the bunching distance being longer than the debunching distance. (2) The target traveling distance is about $40 \mathrm{~cm}$, which may not be long enough for the present condition.

\section{SUMMARY}

We have measured for the first time the CTR from $i 5 \mu \mathrm{m}$ microbunches. The experimental results show that the ATF IFEL wiggler is capable of producing very short bunches at

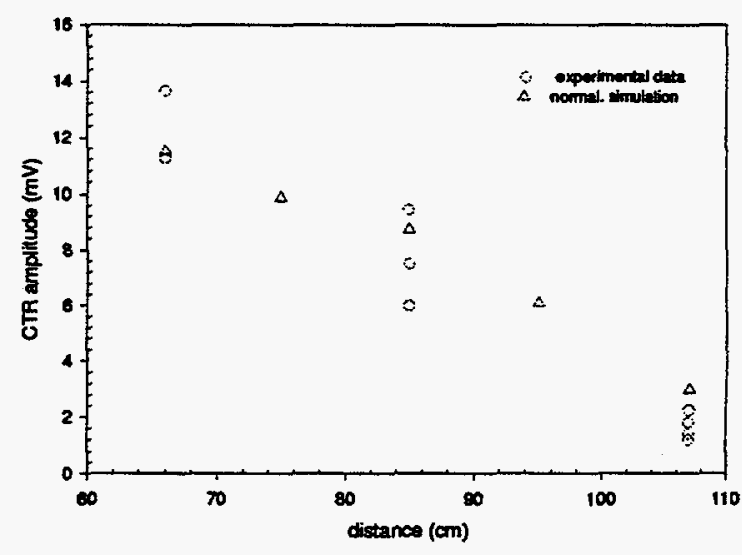

Figure 6: The CTR intensity changes as a function of debunching distance. The circle is the experimental data and the uptriangle is the normalized simulation results.

the several micron level. An experiment to stage two IFEL sections or combine an IFEL section with an ICA section to demonstrate $100 \mathrm{MeV}$ acceleration are in preparation and look very promising.

\section{ACKNOWLEDGEMENTS}

We thank the staff of the ATF at Brookhaven National Laboratory, especially I. Ben-Zvi for his encourage and help, B. Cahill, B. Harrington and M. Montemagno for their technical support. We acknowledge helpful discussions with J. Rosenzweig and E.B. Blum. This work was supported in part by DE-FG03-92ER40695 and DE-AC02$76 \mathrm{CH} 00016$.

\section{REFERENCES}

[1] L.Serafini, IEEE Trans. on Plasma Sci, Vol.24, No. 2, 421 (1996).

[2] T.C. Katsouleas et al, IEEE Trans. on Plasma Sci., Vol.24, No. 2,443 (1996).

[3] A. van Steenbergen, J. Gallardo, J. Sandweiss, J-M. Fang, M. Babzien, X. Qiu, J. Skaritka, and XJ. Wang, Phys. Rev. Lett. 77,2690 (1996).

[4] E.B. Blum, U. Happek and A.J. Sievers, Nucl. Instr. Methods in Phys. Res. A307, 568 (1991).

[5] J. Rosenzweig, G. Travish, and A. Tremaine, Nucl. instr. Methods in Plyys. Res. A365, 255 (1995).

[6] Y. Liu, D.B. Cline, XJ. Wang, M. Babzien, J.M. Fang, and V. Yakimenko, "Micro-bunching Diagnostics for the IFEL by Coherent Transition Radiation" in Proceedings of the Advanced Accelerator Workshop, Lake Tahoe, CA, 12-18 October 1996.

[7] J. Gallardo, "1D IFEL simulation code including space charge". 


\section{DISCLAMER}

This report was prepared as an account of work sponsored by an agency of the United States Government. Neither the United States Government nor any agency thereof, nor any of their employees, makes any warranty, express or implied, or assumes any legal liability or responsibility for the accuracy, completeness, or usefulness of any information, apparatus, product, or process disciosed, or represents that its use would not infringe privately owned rights. Reference herein to any specific commercial product, process, or service by trade name, trademark, manufacturer, or otherwise does not necessarily constitute or imply its endorsement, recommendation, or favoring by the United States Government or any agency thereof. The views and opinions of authors expressed herein do not necessarily state or reflect those of the United States Government or any agency thereof. 


\section{DISCLAMIER}

Portions of this doeument may be illegible in electronic image products. Images are produced from the best avallable original document. 